\title{
EFICIÊNCIA DE SOLUÇÕES DENSAS NO FRACIONAMENTO FÍSICO DA MATÉRIA ORGÂNICA DO SOLO
}

\author{
Paulo Cesar Conceição(1)*, Madalena Boeni ${ }^{(2)}$, Cimélio Bayer $^{(3)}$, Jeferson Dieckow ${ }^{(4)}$, Julio \\ Cesar Salton $^{(5)}$ e Cecilia Estima Sacramento dos Reis ${ }^{(6)}$
}

(1) Universidade Tecnológica Federal do Paraná, Campus Dois Vizinhos, Dois Vizinhos, Paraná, Brasil.

(2) Fundação Estadual de Pesquisa Agropecuária, Centro de Pesquisa de Sementes, Júlio de Castilhos, Rio Grande do Sul.

(3) Universidade Federal do Rio Grande do Sul, Departamento de Solos, Porto Alegre, Rio Grande do Sul, Brasil.

(4) Universidade Federal do Paraná, Departamento de Solos e Engenharia Agrícola, Curitiba, Paraná, Brasil.

(5) Empresa Brasileira de Pesquisa Agropecuária, Centro de Pesquisa Agropecuária do Oeste, Dourados, Mato Grosso do Sul, Brasil.

(6) Universidade Federal do Rio Grande do Sul, Departamento de Solos, Programa de Pós-graduação em Ciência do Solo, Porto Alegre, Rio Grande do Sul, Brasil.

* Autor correspondente.

E-mail: paulocesar@utfpr.edu.br

\section{RESUMO}

Os mecanismos de estabilização da matéria orgânica (MO) têm sido estudados em solos tropicais e subtropicais brasileiros; no entanto, poucos trabalhos avaliaram a influência da parte metodológica do uso das soluções nos resultados obtidos. Objetivou-se avaliar a eficiência de duas soluções salinas (iodeto de sódio - NaI $1,8 \mathrm{~kg} \mathrm{dm}^{-3}$ e politungstato de sódio - PTS $2,0 \mathrm{~kg} \mathrm{dm}^{-3}$ ) na separação de frações densimétricas da MO em dois solos (Argissolo Vermelho do sul do Brasil - $220 \mathrm{~g} \mathrm{~kg}^{-1}$ argila e Latossolo Vermelho do Cerrado - $630 \mathrm{~g} \mathrm{~kg}^{-1}$ argila) e o reflexo dessa eficiência na magnitude e importância do mecanismo de proteção física por oclusão em agregados no acúmulo de MO em solos brasileiros. Amostras de solo coletadas nas camadas de $0,00-0,05 ; 0,05-0,10 ; \mathrm{e} 0,10-0,20 \mathrm{~m}$ foram submetidas ao fracionamento físico densimétrico e separadas as frações leve livre (FLL), leve-oclusa (FLO) e pesada (FP) da MO do solo. O uso de PTS $2,0 \mathrm{~kg} \mathrm{dm}^{-3}$ aumentou o rendimento de carbono orgânico (CO) das FLL e FLO em ambos os solos em relação à solução de $\mathrm{NaI} 1,8 \mathrm{~kg} \mathrm{dm}^{-3}$, sendo o efeito mais pronunciado na FLO. A utilização do sistema plantio direto (PD) aumentou os estoques de CO total na camada de 0,00-0,05 m do Argissolo em relação ao solo em preparo convencional (PC). $\mathrm{O}$ mecanismo de proteção física da MO por oclusão em agregados foi efetivo no aumento dos estoques de $\mathbf{C}$ do solo, sendo responsável por aproximadamente $1 / 3$ do acúmulo na camada superficial do Argissolo (0,00-0,05 m). Para o Latossolo, não houve diferenças entre o estoque 


\begin{abstract}
de $\mathrm{CO}$ total do $\mathrm{PD}$ e do $\mathrm{PC}$, porém o solo sob $\mathrm{PD}$ apresentou acúmulo de aproximadamente $2 / 3$ do estoque de C como FLO na camada superficial do solo. A proteção física por oclusão em agregados é um mecanismo expressivo na estabilização e sequestro de $\mathrm{C}$ em solos tropicais e subtropicais, cuja importância pode ser mascarada pela baixa eficiência da solução de NaI em estudos de fracionamento densimétrico da MO do solo.
\end{abstract}

Palavras-chave: fracionamento densimétrico, dinâmica do carbono orgânico, manejo do solo.

\title{
ABSTRACT: EFFICIENCY OF THE DENSE SOLUTIONS IN PHYSICAL FRACTIONATION OF SOIL ORGANIC MATTER
}

\begin{abstract}
The mechanisms of stabilization of organic matter (OM) have been studied in Brazilian tropical and subtropical soils; however, few studies have evaluated the influence of the methodological part of the use of the solutions in the results. The aim of this study was to evaluate the efficiency of two salt solutions $\left(1.8 \mathrm{~kg} \mathrm{dm}^{-3}\right.$ sodium iodide - NaI, and $2.0 \mathrm{~kg} \mathrm{dm}^{-3}$ sodium polytungstate - SPT) in separation of densimetric soil organic matter (SOM) fractions in two soils (Acrisol from southern Brazil - $220 \mathrm{~g} \mathrm{~kg}^{-1}$ clay, and Ferralsol from the Cerrado [Brazilian tropical savanna] - $630 \mathrm{~g} \mathrm{~kg}^{-1}$ clay) and the impact of this efficiency in interpreting results such as the magnitude and importance of the mechanism of physical protection by occlusion in aggregates in organic matter accumulation in Brazilian soils. Soil samples taken from the 0.00-0.05, 0.05-0.10, and 0.10-0.20 m layers underwent density fractionation, and the free light fraction $(F L F)$, occluded light fraction $(O L F)$, and heavy fraction $(\mathrm{HF})$ of OM were obtained. The use of $2.0 \mathrm{~kg} \mathrm{dm}^{-3}$ SPT increased the content of organic carbon (C) in the FLF and in the OLF of both soils in comparison to $1.8 \mathrm{~kg} \mathrm{dm}^{-3} \mathrm{NaI}$, and the effect was more pronounced in the OLF. Use of the NT system increased the SOM content in the 0-0.05 m layer compared to CT in the Acrisol. The mechanism of physical protection of SOM by occlusion in aggregates was effective in increasing soil C stocks, accounting for $1 / 3$ of $C$ accumulation in the 0.00-0.05 m layer of the Acrisol. For the Ferralsol, there were no differences between the stock of total organic carbon (TOC) in NT and CT, but the soil in NT showed an accumulation of approximately $2 / 3$ of the $C$ stock as OLF in the topsoil. Physical protection by occlusion in aggregates is a significant mechanism in $C$ stabilization and sequestration in tropical and subtropical soils, but its importance may be masked by the low efficiency of the NaI solution in studies of densimetric fractionation of SOM.
\end{abstract}

Keywords: densimetric fractionation, soil organic carbon dynamic, soil management.

\section{INTRODUÇÃO}

A utilização do fracionamento físico densimétrico em estudos da matéria orgânica do solo (MO) se tornou comum na ciência do solo internacional na última década (Diochon e Kellman, 2009; Shi et al., 2010; Paré e Bedard-Haughn, 2011; Conceição et al., 2013) e também nas pesquisas brasileiras (Roscoe et al., 2001; Freixo et al., 2002a,b; Conceição et al., 2008, 2014). Essa metodologia possibilita a separação de compartimentos com diferentes dinâmicas e funções no solo (Moni et al., 2012), permitindo analisar a relação entre a composição da matéria orgânica (MO) e a sua localização na estrutura do solo, bem como a sua interação com a matriz mineral (Christensen, 1992). O fracionamento densimétrico baseia-se na separação das frações orgânica e mineral do solo, mediante uso de soluções com densidades variando de 1,1 a $2,4 \mathrm{~kg} \mathrm{dm}^{-3}$ (Christensen, 1992). Soluções inorgânicas predominam em estudos de fracionamento densimétrico da MO, com destaque atualmente para as soluções de iodeto de sódio (NaI) e de politungstato de sódio (PTS), basicamente em razão da sua menor toxidez em comparação às soluções orgânicas inicialmente empregadas (Christensen, 1992; Morgun e Makarov, 2011). O uso de NaI permite obter soluções, em temperatura ambiente, com densidade até aproximadamente $1,85 \mathrm{~kg} \mathrm{dm}^{-3}$, enquanto o uso de PTS possibilita obter soluções com densidades de até $3,0 \mathrm{~kg} \mathrm{dm}^{-3}$ (Morgun e Makarov, 2011)

O fracionamento densimétrico clássico admite a obtenção de três frações da MO (Sollins et al., 1996), as quais distinguem-se, em relação à sua dinâmica e estabilidade, quanto à sua localização na estrutura do solo e ao grau de interação com a matriz mineral, particularmente com a fração mais fina. A fração leve livre (FLL) ou interagregados possui composição molecular comparável àquela de materiais vegetais dos quais derivaram (Freixo et al., 2002a), e o principal mecanismo de proteção atuante é a recalcitrância intrínseca da biomolécula (Sollins et al., 1996; Kleber et al., 2011). A fração leve oclusa (FLO) ou intra-agregados compreende um diversificado conjunto de compostos orgânicos com grau de decomposição geralmente mais avançado em 
comparação à FLL (Golchin et al., 1994a,b; Freixo et al., 2002a; Wagai et al., 2009), e os mecanismos de estabilização atuantes são a recalcitrância molecular e, principalmente, a proteção física por oclusão em agregados de solo (Sollins et al., 1996). A fração pesada (FP) é composta basicamente pela MO associada aos minerais do solo (Kaiser et al., 2007), apresentando estágio mais avançado de decomposição (Christensen, 1992; Sollins et al., 1996). Nessa fração, em adição ao mecanismo de recalcitrância e oclusão, ocorre a estabilidade coloidal da MO pela forte interação com a matriz mineral, conferindo a esse compartimento a maior contribuição para o armazenamento de carbono orgânico (CO) no solo (von Lützow e Kögel-Knabner, 2009).

Embora o uso corrente do NaI e do PTS no fracionamento físico densimétrico (Sohi et al., 2001; Freixo et al., 2002b; Diekow et al., 2005; Basile-Doelsch et al., 2009; Hofmockel et al., 2011; Potes et al., 2012), poucos estudos têm testado a eficiência de soluções e densidades elaboradas com o uso desses produtos quanto à quantificação das frações da MO. Golchin et al. (1994b) preconizaram o uso de solução de PTS $1,6 \mathrm{~kg} \mathrm{dm}^{-3}$, tendo obtidos resultados com predominância da FLL sobre a FLO. Parfitt et al. (1997) utilizaram PTS $1,6 \mathrm{~kg} \mathrm{dm}^{-3} \mathrm{e}$, de forma diferente dos resultados de Golchin et al. (1994b), obtiveram maiores rendimentos de $\mathrm{C}$ na FLO em relação à FLL. Entretanto, o uso de PTS 1,8 e 2,0 $\mathrm{kg} \mathrm{dm}^{-3}$ resultou em aumento do rendimento de C da FLO, sendo superior ao rendimento obtido para a FLL nos estudos de Golchin et al. (1994a). Sohi et al. (2001), avaliando o efeito da densidade da solução no rendimento de $\mathrm{C}$, propuseram a utilização de densidade de $1,8 \mathrm{~kg} \mathrm{dm}^{-3}$ como ideal para a solução de NaI. Freixo et al. (2002a,b), utilizando $\mathrm{NaI} 1,8 \mathrm{~kg} \mathrm{dm}^{-3}$, obtiveram rendimento de C na FLO inferiores aos obtidos para a FLL, similar ao ocorrido em estudos desenvolvidos por Roscoe et al. (2001).

A maior recuperação de C com solução de PTS $2,0 \mathrm{~kg} \mathrm{dm}^{-3}$ não é efeito exclusivo da maior densidade da solução, pois, mesmo com o uso de PTS $1,8 \mathrm{~kg} \mathrm{dm}^{-3}$, ocorrem incrementos de $\mathrm{C}$ que variam de 176 a $726 \%$, em comparação à solução de $\mathrm{NaI} 1,8 \mathrm{~kg} \mathrm{dm}^{-3}$ (Conceição et al., 2008). Cerli et al. (2012) também verificaram valores similares para a recuperação de $\mathrm{C}$ nas frações físicas pelo uso de solução de PTS com densidades de 1,8 e $2,0 \mathrm{~kg} \mathrm{dm}^{-3}$. Estudos sobre proteção física da MO em Latossolos brasileiros (Roscoe et al., 2001; Freixo et al., 2002b) também revelaram maiores concentrações de $\mathrm{C}$ na FLL em comparação à FLO, em decorrência do uso da solução de $\mathrm{NaI} 1,8 \mathrm{~kg} \mathrm{dm}^{-3}$. Nesses estudos, esse resultado pode ser atribuído a dois fatores: possível interação entre o íon iodeto $\left(\mathrm{I}^{-}\right)$e a $\mathrm{MO}$ presente na FLO; e uso de solo moído a $2 \mathrm{~mm}$. A menor capacidade da solução de NaI $1,8 \mathrm{~kg} \mathrm{dm}^{-3} \mathrm{em}$ recuperar FLO pode ser explicada pela associação entre I' e os grupos funcionais orgânicos (Schlegel et al., 2006), pois essa fração apresenta maior grau de decomposição comparativamente à FLL (Sohi et al., 2001). Embora existam evidências dos mecanismos envolvidos na retenção de I- à matéria orgânica (Johanson, 2000; Dai et al., 2009), seus efeitos no processo de fracionamento da MO ainda não são totalmente conhecidos. Estudo desenvolvido por Moulin et al. (2001) revelou que a associação entre compostos de iodo e estruturas da MO ocorre rapidamente quando a concentração de iodo em solução é alta e depende do estágio de humificação da MO, possivelmente pela presença de grupamentos orgânicos onde o iodo se associa preferencialmente como os compostos aromáticos (Radlinger e Heumann, 2000; Schlegel et al., 2006, Yamaguchi et al. 2010). A utilização de solo moído a $2 \mathrm{~mm}$ também aumenta a proporção da FLL em razão da liberação de material orgânico ocluso em agregados maiores que $2 \mathrm{~mm}$ (Tomazi et al., 2011). Portanto, a moagem parcial $(2 \mathrm{~mm})$ de solo pode explicar alguns resultados existentes na literatura, em que as evidências da proteção física da MO não são tão explícitas. Nesse sentido, Tomazi et al. (2011) destacaram que, em estudos onde o objetivo é avaliar a proteção física da MO, os agregados de solo devem ser preservados a partir da utilização de amostras de 8,00 ou $9,51 \mathrm{~mm}$.

Dessa forma, os objetivos deste estudo foram avaliar a eficiência de duas soluções salinas (iodeto de sódio - NaI $1,8 \mathrm{~kg} \mathrm{dm}^{-3}$ e politungstato de sódio - PTS 2,0 $\mathrm{kg} \mathrm{dm}^{-3}$ ) na separação de frações densimétricas da MO em dois solos (Argissolo Vermelho do sul do Brasil - $220 \mathrm{~g} \mathrm{~kg}^{-1}$ argila e Latossolo Vermelho do Cerrado - $630 \mathrm{~g} \mathrm{~kg}^{-1}$ argila) e o reflexo dessa eficiência na magnitude e importância do mecanismo de proteção física por oclusão em agregados no acúmulo de MO em solos brasileiros.

\section{MATERIAL E MÉTODOS}

O estudo foi desenvolvido com base em amostras de solo de dois experimentos de campo de longa duração em solos distintos. O primeiro está localizado na Estação Experimental Agronômica da Universidade Federal do Rio Grande do Sul $\left(30^{\circ} 50^{\prime} \mathrm{S}, 51^{\circ} 38^{\prime} \mathrm{O}\right)$ em Eldorado do Sul, RS, instalado em 1985 sobre um Argissolo Vermelho distrófico típico (Embrapa, 2013) com textura franco-argiloarenosa (220 $\mathrm{g} \mathrm{kg}^{-1}$ de argila), razão gibsita/(gibsita + caulinita) $(0,02)$ e $56 \mathrm{~g} \mathrm{~kg}^{-1} \mathrm{de}$ $\mathrm{Fe}_{2} \mathrm{O}_{3}$. O clima da região, segundo classificação de Köppen (Alvares et al., 2013), é subtropical de verão úmido e quente, do tipo $\mathrm{Cfa}$, com temperatura média anual de $19{ }^{\circ} \mathrm{C}$ e precipitação pluvial média anual de $1.440 \mathrm{~mm}$. O segundo, instalado em 1995, localiza-se na área experimental da Embrapa Agropecuária Oeste (22 $2^{\circ} 14^{\prime} \mathrm{S}, 54^{\circ} 49^{\prime}$ 0) em Dourados, MS, sob clima tropical e em um Latossolo 
Vermelho distroférrico (Embrapa, 2013), com textura muito argilosa (630 $\mathrm{g} \mathrm{kg}^{-1}$ de argila), razão gibsita/(gibsita + caulinita) $(0,13)$ e $135 \mathrm{~g} \mathrm{~kg}^{-1} \mathrm{de}$ $\mathrm{Fe}_{2} \mathrm{O}_{3}$ (Inda Junior et al., 2007). O clima da região, segundo classificação de Köppen (Alvares et al., 2013), é tropical (Aw) com estação quente e chuvosa no verão e seca no inverno, com temperatura média anual do ar de $23{ }^{\circ} \mathrm{C}$ e precipitação pluvial média anual de $1.635 \mathrm{~mm}$.

Em ambos os solos, a amostragem foi realizada em 2004, quando os experimentos apresentavam 18 anos (sul do Brasil) e nove anos (centro-oeste). A amostragem de solo foi realizada em preparo convencional (PC) e em plantio direto (PD). No Argissolo, o sistema de culturas consistia no cultivo de aveia-preta (Avena strigosa) + ervilhaca (Vicia sativa) no inverno e milho (Zea mays) + caupi (Vigna unguiculata), no verão. No Latossolo, o trigo (triticum aestivum) foi cultivado no inverno; e a soja (Glicine max), no verão.

Foram abertas trincheiras de $0,20 \times 0,40 \mathrm{~m}$ transversais às linhas de semeadura. Coletaram-se as amostras nas profundidades de 0,00-0,05; 0,05-0,10; e 0,10-0,20 m com auxílio de espátula, as quais foram secas ao ar, moídas manualmente com rolo e passadas em peneira com malha de $2 \mathrm{~mm}$. $\mathrm{O}$ teor de carbono orgânico total (COT) foi obtido por combustão seca (Shimadzu-TOC-V CSH) nas amostras moídas em gral e passadas em malha de $250 \mu \mathrm{m}$. Nessas profundidades, também foram coletados blocos indeformados de $0,20 \times 0,10 \mathrm{~m}$, separados os agregados do solo manualmente a partir dos seus principais planos de fraqueza, passados em peneira com malha de $9,51 \mathrm{~mm}$ e secos ao ar. Para obter FLL e FLO (Golchin et al., 1994b), $10 \mathrm{~g}$ de agregados foram colocados em tubo de centrífuga de $100 \mathrm{~mL}$, contendo $80 \mathrm{~mL}$ de solução de NaI $1,8 \mathrm{~kg} \mathrm{dm}^{-3}$ ou PTS $2,0 \mathrm{~kg} \mathrm{dm}^{-3}$, cujo $\mathrm{pH}$ foi mantido em 4,0, a fim de evitar a solubilização de MO. O tubo foi fechado com rolha e invertido lenta e manualmente por cinco vezes, evitando o rompimento dos agregados, visando à liberação da FLL (Golchin et al., 1994a). A suspensão foi centrifugada a $2.000 \mathrm{~g}$ por 90 min e o sobrenadante contendo a FLL filtrado sob vácuo, em filtro Whatman GF/C. Para retirar o excesso de sal (NaI ou PTS), o filtro+FLL foi lavado com água destilada, seco a $60{ }^{\circ} \mathrm{C}$ por $24 \mathrm{~h}$ e quantificada a massa de filtro+FLL. Para separar a FLO, a solução de NaI ou PTS foi retornada ao tubo contendo o pellet que foi ressuspenso e submetido à dispersão por ultrassom, com energia necessária para a máxima dispersão do solo em partículas primárias $\left(250 \mathrm{~J} \mathrm{~mL}^{-1}\right.$ para o Argissolo e $450 \mathrm{~J} \mathrm{~mL}^{-1}$ para o Latossolo), de acordo com Inda Junior et al. (2007). Após a dispersão, a suspensão foi centrifugada a $2.000 \mathrm{~g}$ por $90 \mathrm{~min}$; e a FLO filtrada, da mesma forma que a FLL.

As FLL e FLO foram analisadas em relação aos teores de C por combustão seca (Shimadzu-TOC-V
$\mathrm{CSH}$ ), sendo o C da fração pesada (FP) obtido por diferença entre o COT e a soma dos teores de $\mathrm{C}$ das FLL e FLO. Os estoques de C no solo inteiro e nas frações da MO foram calculados em massa equivalente, conforme proposto por Ellert e Bettany (1995), considerando a massa de solo de uma área sob vegetação nativa adjacente ao experimento como referência, sendo sob cerrado, em Dourados, e sob campo nativo, no RS.

Os valores de estoques de COT do solo e de $\mathrm{C}$ das frações físicas da MO foram submetidos à análise da variância, considerando um delineamento em blocos ao acaso, com três repetições, para cada área experimental, sendo as diferenças entre médias avaliadas pelo teste de Tukey a $5 \%$. Os coeficientes de correlação foram obtidos por meio da comparação entre os dados com uso de NaI e PTS, para ambos os solos.

\section{RESULTADOS E DISCUSSÃO}

A baixa eficiência da solução de $\mathrm{NaI}$ em recuperar as frações da MO foi evidenciada pelas regressões lineares entre as concentrações de C da FLL e FLO, obtidas com soluções de NaI e PTS (Figura 1). Em comparação à solução de $\mathrm{NaI} 1,8 \mathrm{~kg} \mathrm{dm}^{-3}$, a utilização da solução de PTS resultou em incremento no rendimento de $\mathrm{C}$ da FLL na ordem de 1,6 vez para o Argissolo (Figura 1a), enquanto o incremento para a FLO foi da ordem de 6,8 vezes (Figura 1a). Portanto, a subestimação do rendimento de $\mathrm{C}$, como resultado do uso de solução de NaI, foi maior na FLO do que na FLL. Para o Latossolo (Figura 1b), esse efeito foi ainda mais pronunciado, visto que, com o uso de solução de PTS, houve rendimento de carbono 8,7 vezes superior na FLO em relação ao uso de solução de NaI. Para a FLL, o incremento de $\mathrm{C}$ pelo uso de PTS foi de apenas 1,3 vez.

A maior recuperação de C na FLO em relação à FLL, verificada com a utilização da solução de PTS (Figura 1, Quadro 1), resultou em estoques superiores da FLO em relação à FLL. Para as camadas de 0,05-0,10 e 0,10-0,20 m, o rendimento de C da FLO foi superior ao da FLL, mesmo com o uso de solução de NaI. Considerando-se as três profundidades avaliadas e os dois tratamentos, os dois solos apresentaram, em média, baixa porcentagem de COT protegido em ambas as frações (2,3 \% na FLL e $2,1 \%$ na FLO), a partir do uso de NaI. Freixo et al. (2002a,b) também verificaram baixa contribuição da FLL $(4,2 \%)$ e da $\operatorname{FLO}(0,7 \%)$ para o COT em Latossolos tropicais e subtropicais com o uso de solução de NaI. No entanto, com o uso de solução de PTS, a média foi de 4,0 e 15,8 \% do COT na FLL e FLO, respectivamente (Quadro 1). Dessa forma, ocorreu expressivo aumento na recuperação de C na FLO em razão do uso de solução de PTS, 


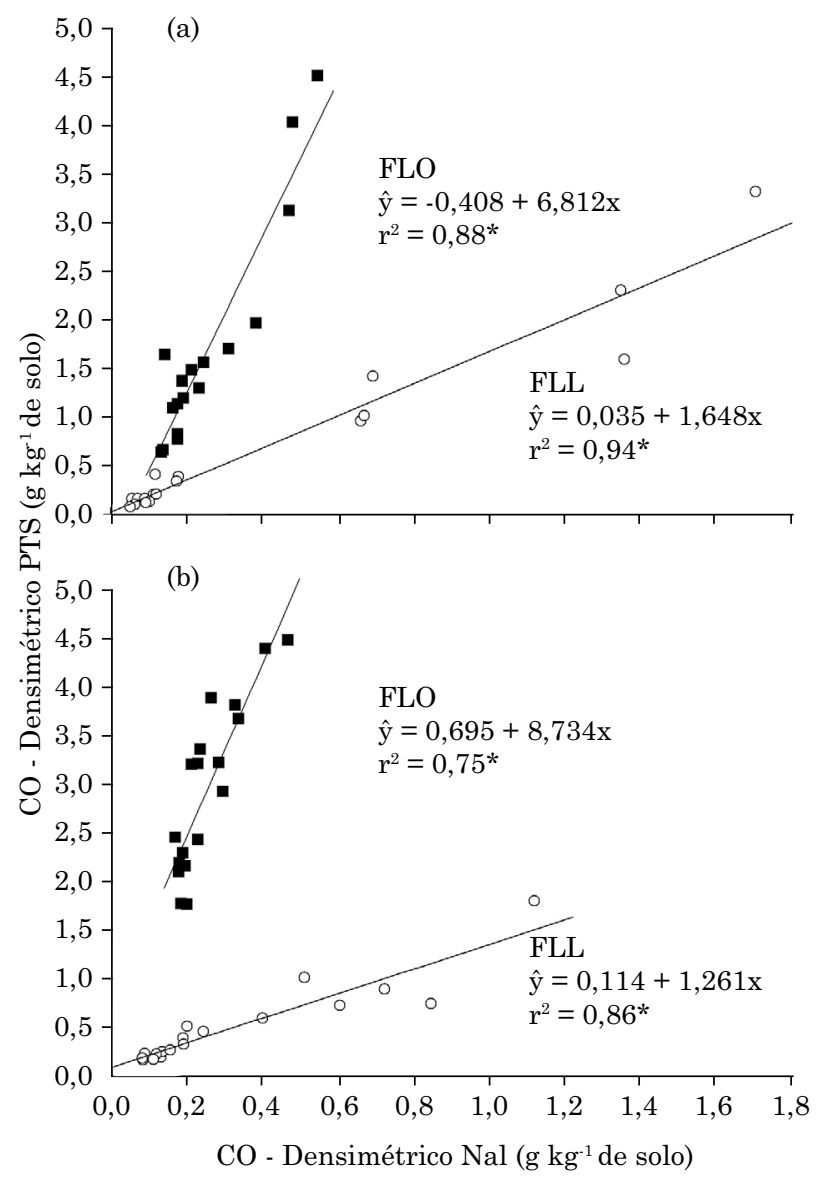

Figura 1. Rendimento de carbono orgânico obtido com as soluções de iodeto de sódio ( $\mathrm{NaI} 1,8 \mathrm{~kg} \mathrm{dm}^{-3}$ ) e politungstato de sódio (PTS $2,0 \mathrm{~kg} \mathrm{dm}^{-3}$ ) das frações leve livre (FLL) e leve oclusa (FLO) da matéria orgânica de um Argissolo Vermelho do sul do Brasil (a) e de um Latossolo Vermelho do Cerrado (b). * significativo a p $<0,0001$.

ressaltando a importância do mecanismo de proteção por oclusão (FLO). Os maiores percentuais na FP são condizentes com a estabilidade química da MO associada aos minerais, pois essa fração possuiu efeito de três mecanismos de proteção. Dessa maneira, a importância da FP obtida com PTS (63 a $89 \%$ do COT) foi menor em comparação à FP obtida com solução de $\mathrm{NaI}$ (88 a $98 \%$ ), pois nesse caso a separação da FLL e FLO pode não ter ocorrido de maneira eficiente no processo sequencial do fracionamento densimétrico.

No Argissolo, no fracionamento da MO utilizando a solução de $\mathrm{NaI}$ foi verificado o efeito do manejo de solo em $\mathrm{PD}$ na proteção da MO na camada de 0,00-0,05 m, com o estoque de C da FLL e FP do solo em PD significativamente superior aos obtidos para o solo em PC. Por sua vez, no uso da solução de PTS foi averiguado que a FLO da camada de 0,00-0,05 m do Argissolo em PD apresentou maior estoque de C comparativamente ao solo sob PC, enquanto a FP não variou. Tal resultado é decorrente da maior adição de resíduos em superfície (Vieira et al., 2009; Sun et al., 2011) e melhor agregação das partículas do solo, quando é empregado um sistema conservacionista de manejo (Conceição et al., 2008), enquanto, sob PC, o revolvimento do solo causa o rompimento dos agregados e, consequentemente, exposição da $\mathrm{MO}$ aos microrganismos e suas enzimas (Shi et al., 2010).

No Latossolo, o uso de solução de $\mathrm{NaI}$ não diferenciou os sistemas de manejo entre si para as frações. À semelhança do que foi verificado no Argissolo, com o uso de solução de PTS, o estoque de $\mathrm{C}$ na FLO foi superior no solo em PD, em comparação ao solo em PC. Para ambos os solos, o estoque de C da FLL foi diferente entre os sistemas de preparo na camada de 0,05-0,10 m com o uso de solução de PTS. Esse comportamento na segunda camada amostrada, favorável ao solo em PC, pode ter sido decorrente da incorporação e redistribuição de resíduos orgânicos (Rumpel et al., 2011) promovidas pelos equipamentos de preparo do solo no PC, enquanto, no PD, os resíduos foram mantidos em superfície.

Analisando a magnitude de proteção da MO nas frações de ambos os solos com o uso de solução de PTS, foi verificado um gradiente crescente de estoque $(\mathrm{FLL}<\mathrm{FLO}<\mathrm{FP})$ em todas as camadas, coerente com os mecanismos de estabilização atuantes nas respectivas frações (Sollins et al., 1996; Conceição et al., 2008, 2013). Tal comportamento não foi observado quando da utilização da solução de NaI, nem mesmo na camada superficial do solo (0,00-0,05 m), a qual usualmente apresenta os maiores acúmulos de $\mathrm{MO}$ no solo em $\mathrm{PD}$, sendo a FLO inferior às FLL.

O estoque de COT na camada de 0,00-0,20 m do Argissolo foi superior no solo em PD (37,3 $\left.\mathrm{Mg} \mathrm{ha}^{-1}\right)$, em comparação ao PC $\left(32,8 \mathrm{Mg} \mathrm{ha}^{-1}\right)$ (Quadro 1). Para o Latossolo, não houve diferença entre os estoques de COT em PD (40,3 $\left.\mathrm{Mg} \mathrm{ha}^{-1}\right)$ e em PC $\left(40,9 \mathrm{Mg} \mathrm{ha}^{-1}\right)$, evidenciando a maior dificuldade no aumento da $\mathrm{MO}$ em solos argilosos em PD, quando da utilização de sistemas de cultura com baixas adições de resíduos. Considerando que o efeito da proteção física da MO foi mais efetivo na camada de 0,00-0,05 m (Quadro 2), foi calculada a variação dos estoques das frações e do solo integral entre o solo em PD e o em PC (Quadro 2). Para o Argissolo, o uso do solo em PD aumentou o estoque de COT em $5,7 \mathrm{Mg} \mathrm{ha}^{-1}$ em relação ao solo em PC, enquanto para o Latossolo a variação foi de apenas $0,6 \mathrm{Mg} \mathrm{ha}^{-1} \mathrm{na}$ mesma comparação. Quando a solução utilizada foi o NaI, cerca de 11 e $23 \%$ da variação do COT foram em razão dos mecanismos de recalcitrância (FLL) para o Argissolo e o Latossolo, respectivamente (Quadro 2). Para o Argissolo, apenas $4 \%$ da variação do estoque de COT entre o solo em PC e o em PD foi por causa da proteção por oclusão, enquanto, no Latossolo, $11 \%$ do COT foram protegidos por esse 
Quadro 1. Estoque da fração leve livre (FLL), leve oclusa (FLO) e pesada (FP) da matéria orgânica do solo obtido com solução de iodeto de sódio (NaI) e politungstato de sódio (PTS) em dois solos manejados sob preparo convencional (PC) e plantio direto (PD)

\begin{tabular}{|c|c|c|c|c|c|c|c|c|}
\hline \multirow{2}{*}{$\begin{array}{l}\text { Sistema de } \\
\text { preparo }\end{array}$} & \multirow[t]{2}{*}{ Prof. } & \multicolumn{3}{|c|}{$\mathrm{NaI} 1,8 \mathrm{~kg} \mathrm{dm}^{-3}$} & \multicolumn{3}{|c|}{ PTS $2,0 \mathrm{~kg} \mathrm{dm}^{-3}$} & \multirow[t]{2}{*}{ COT } \\
\hline & & FLL & FLO & FP & FLL & FLO & FP & \\
\hline & \multirow[t]{2}{*}{$\mathrm{m}$} & \multicolumn{7}{|c|}{$\mathrm{Mg} \mathrm{ha}^{-1}$} \\
\hline \multicolumn{8}{|c|}{ Argissolo Vermelho } & \\
\hline $\mathrm{PC}$ & $0,00-0,05$ & $0,50 \mathrm{~b}$ & $0,29 \mathrm{a}$ & $7,65 \mathrm{~b}$ & $0,86 \mathrm{a}$ & $1,58 \mathrm{~b}$ & $6,01 \mathrm{a}$ & $8,45 \mathrm{~b}$ \\
\hline \multirow[t]{2}{*}{$\mathrm{PD}$} & & $1,10 \mathrm{a}$ & $0,52 \mathrm{a}$ & $12,51 \mathrm{a}$ & $1,81 \mathrm{a}$ & $3,46 \mathrm{a}$ & $8,87 \mathrm{a}$ & $14,14 \mathrm{a}$ \\
\hline & $\mathrm{CV}(\%)$ & 13,6 & 18,5 & 11,5 & 35,8 & 15,1 & 14,8 & 10,7 \\
\hline $\mathrm{PC}$ & $0,05-0,10$ & $0,12 \mathrm{a}$ & $0,23 \mathrm{a}$ & $7,92 \mathrm{a}$ & $0,31 \mathrm{a}$ & $1,34 \mathrm{a}$ & $6,63 \mathrm{a}$ & $8,27 \mathrm{a}$ \\
\hline \multirow[t]{2}{*}{$\mathrm{PD}$} & & $0,07 \mathrm{a}$ & $0,21 \mathrm{a}$ & $8,10 \mathrm{a}$ & $0,15 \mathrm{~b}$ & $1,18 \mathrm{a}$ & $7,05 \mathrm{a}$ & $8,37 \mathrm{a}$ \\
\hline & CV (\%) & 21,8 & 20,0 & 14,1 & 10,9 & 15,1 & 18,3 & 13,6 \\
\hline $\mathrm{PC}$ & $0,10-0,20$ & $0,15 \mathrm{a}$ & $0,45 \mathrm{a}$ & 15,48 & $0,28 \mathrm{a}$ & $2,11 \mathrm{a}$ & 13,68 a & $16,07 \mathrm{a}$ \\
\hline \multirow[t]{2}{*}{$\mathrm{PD}$} & & $0,11 \mathrm{a}$ & $0,34 \mathrm{a}$ & $14,30 \mathrm{a}$ & $0,18 \mathrm{a}$ & $1,36 \mathrm{a}$ & $13,21 \mathrm{a}$ & $14,75 \mathrm{a}$ \\
\hline & CV (\%) & 22,0 & 20,4 & 14,1 & 24,4 & 24,3 & 15,1 & 14,0 \\
\hline \multicolumn{9}{|c|}{ Latossolo Vermelho } \\
\hline $\mathrm{PC}$ & $0,00-0,05$ & $0,30 \mathrm{a}$ & $0,15 \mathrm{a}$ & $10,19 \mathrm{a}$ & $0,40 \mathrm{a}$ & $1,76 \mathrm{~b}$ & $8,49 \mathrm{a}$ & $10,64 \mathrm{a}$ \\
\hline \multirow[t]{2}{*}{ PD } & & $0,43 \mathrm{a}$ & $0,21 \mathrm{a}$ & $10,56 \mathrm{a}$ & $0,63 \mathrm{a}$ & $2,18 \mathrm{a}$ & $8,40 \mathrm{a}$ & $11,21 \mathrm{a}$ \\
\hline & CV (\%) & 30,2 & 20,6 & 12,5 & 29,3 & 5,2 & 14,8 & 11,0 \\
\hline $\mathrm{PC}$ & $0,05-0,10$ & $0,11 \mathrm{a}$ & $0,12 \mathrm{a}$ & $9,44 \mathrm{a}$ & $0,24 \mathrm{a}$ & $1,74 \mathrm{a}$ & $7,69 \mathrm{a}$ & 9,67 a \\
\hline \multirow[t]{2}{*}{ PD } & & $0,08 \mathrm{a}$ & $0,12 \mathrm{a}$ & $9,03 \mathrm{a}$ & $0,15 \mathrm{~b}$ & $1,32 \mathrm{~b}$ & $7,76 \mathrm{a}$ & $9,23 \mathrm{a}$ \\
\hline & CV (\%) & 19,4 & 5,5 & 3,2 & 7,4 & 4,5 & 3,6 & 3,2 \\
\hline $\mathrm{PC}$ & $0,10-0,20$ & $0,12 \mathrm{a}$ & $0,22 \mathrm{a}$ & $20,25 \mathrm{a}$ & $0,27 \mathrm{a}$ & $2,81 \mathrm{a}$ & $17,51 \mathrm{a}$ & $20,59 \mathrm{a}$ \\
\hline \multirow[t]{2}{*}{ PD } & & $0,13 \mathrm{a}$ & $0,23 \mathrm{a}$ & 19,49 a & $0,26 \mathrm{a}$ & $2,29 \mathrm{~b}$ & $17,30 \mathrm{a}$ & $19,85 \mathrm{a}$ \\
\hline & CV (\%) & 16,9 & 5,0 & 3,3 & 6,7 & 2,6 & 3,3 & 3,2 \\
\hline
\end{tabular}

Médias seguidas pela mesma letra na coluna comparam sistemas de preparo dentro de cada profundidade para cada solo separadamente, não sendo diferentes entre si pelo teste de Tukey a $5 \%$.

Quadro 2. Variação $(\Delta)$ do estoque de carbono orgânico total (COT) e de C das frações leve livre (FLL), leve oclusa (FLO) e pesada (FP) da matéria orgânica do solo, obtida com solução de iodeto de sódio (NaI) e politungstato de sódio (PTS), entre os sistemas de preparo convencional (PC) e plantio direto (PD) para a camada de 0,00-0,05 m, e relação da variação do estoque de carbono das frações $\left(\Delta \mathrm{C}_{\text {fração }}\right)$ com a variação do estoque de carbono orgânico total ( $\triangle$ COT), entre os sistemas de preparo

\begin{tabular}{|c|c|c|c|c|c|c|c|}
\hline \multirow[t]{2}{*}{ Solução } & \multicolumn{4}{|c|}{$\Delta \mathrm{C}(\mathrm{PD}-\mathrm{PC})^{(1)}$} & \multicolumn{3}{|c|}{$\Delta \mathbf{C}_{\text {fração }} / \Delta \mathbf{C - C O T}$} \\
\hline & C FLL & C FLO & C FP & COT & FLL & FLO & FP \\
\hline & \multicolumn{4}{|c|}{$\mathrm{Mg} \mathrm{ha}^{-1}$} & & & \\
\hline & \multicolumn{7}{|c|}{ Argissolo Vermelho } \\
\hline $\mathrm{NaI}$ & 0,60 & 0,23 & 4,86 & & 11 & 4 & 85 \\
\hline \multirow[t]{2}{*}{ PTS } & 0,95 & 1,88 & 2,86 & 5,69 & 17 & 33 & 50 \\
\hline & \multicolumn{7}{|c|}{ Latossolo Vermelho } \\
\hline $\mathrm{NaI}$ & 0,13 & 0,06 & 0,38 & & 23 & 11 & 66 \\
\hline PTS & 0,23 & 0,42 & $-0,09$ & 0,57 & 42 & 75 & -17 \\
\hline
\end{tabular}

(1) $\Delta$ = estoque de $\mathrm{C}$ do solo em PD - estoque de $\mathrm{C}$ do solo em PC. 
mecanismo (Quadro 2). A importância da oclusão como mecanismo de proteção da $\mathrm{MO}$ e do aumento do estoque de $\mathrm{C}$ do solo em $\mathrm{PD}$ foi verificada pela maior proporção de C na FLO, quando a solução utilizada foi a de PTS. Para o Argissolo, um terço do incremento no estoque de $\mathrm{C}$ no solo em $\mathrm{PD}$ em relação ao solo em PC ocorreu na FLO, enquanto no Latossolo essa proporção atingiu 75 \% (Quadro 2). Assim, com o uso de solução de NaI, a FLO foi a fração que menos acumulou $\mathrm{C}$ entre os sistemas de preparo do solo, enquanto com o uso de solução de PTS essa fração passou a ter importância similar ou até superior à proteção da MO decorrente da interação com a fração mineral do solo.

Dessa forma, estoques de $\mathrm{C}$ em solos brasileiros podem decorrer da oclusão em agregados e da interação da MO com a fração mineral do solo, que são os dois principais mecanismos propostos para a estabilização da MO. Ao utilizar soluções de $\mathrm{NaI}$, os efeitos da oclusão podem ser subestimados, enquanto em estudos com o uso de solução de PTS a maior eficiência desse produto em recuperar frações orgânicas permite obter resultados coerentes com a teoria dos mecanismos de proteção da MO atualmente aceita.

\section{CONCLUSÕES}

A importância do mecanismo de proteção física por oclusão em agregados pode ser subestimada pelos tipos de solução utilizada no fracionamento densimétrico da matéria orgânica.

$\mathrm{O}$ uso de solução de $\mathrm{NaI} 1,8 \mathrm{~kg} \mathrm{dm}^{-3}$ subestima o rendimento de $\mathrm{C}$ das frações leves da matéria orgânica, sendo esse efeito mais pronunciado na fração leve oclusa do que na fração leve livre, possivelmente em razão da interação do íon iodeto com a matéria orgânica presente na fração leve oclusa.

Aproximadamente um terço do acúmulo de $\mathrm{C}$ orgânico verificado na camada superficial $(0,00-0,05 \mathrm{~m})$ dos solos em plantio direto avaliados deve-se à sua estabilização por oclusão em agregados de solo.

\section{REFERÊNCIAS}

Alvares CA, Stape JL, Sentelhas PC, Gonçalves JLM, Sparovek G. Köppen's climate classification map for Brazil. Meteorol Zeitschrift. 2013;22:711-28.

Basile-Doelsch I, Brun T, Borschneck D, Masion A, Marol C, Balesdent J. Effect of land use on organic matter stabilized in organo-mineral complexes: A study combining density fractionation, mineralogy and $\delta^{13} \mathrm{C}$. Geoderma. 2009;151:77-86.

Cerli C, Celi L, Kalbitz K, Guggenberger G, Kaiser K. Separation of light and heavy organic matter fractions in soil - Testing for proper density cut-off and dispersion level. Geoderma. 2012;170:403-16.

Christensen BT. Physical fractionation of soil and organic matter in primary particle size and density separates. Adv Soil Sci. 1992;20:1-90.

Conceição PC, Boeni M, Dieckow J, Bayer C, Mielniczuk J. Fracionamento densimétrico com politungstato de sódio no estudo da proteção física da matéria orgânica em solos. R Bras Ci Solo. 2008;32:541-9.

Conceição PC, Dieckow J, Bayer C. Combined role of no-tillage and cropping systems in soil carbon stocks and stabilization. Soil Till Res. 2013;129:40-7.

Conceição PC, Bayer C, Dieckow J, Santos DC. Fracionamento físico da matéria orgânica e índice de manejo de carbono de um Argissolo submetido a sistemas conservacionistas de manejo. $\mathrm{Ci}$ Rural. 2014;44:794-800.

Dai JL, Zhang M, Hu QH, Huang YZ, Wang RQ, Zhu YG. Adsorption and desorption of iodine by various Chinese soils: II. Iodide and iodate. Geoderma. 2009;153:130-5.

Diekow J, Mielniczuk J, Knicker H, Bayer C, Dick DP, Kögel-Knabner I. Carbon and nitrogen stocks in physical fractions of a subtropical Acrisol as influenced by long-term no-till cropping systems and N fertilization. Plant Soil. 2005;268:319-28.

Diochon AC, Kellman L. Physical fractionation of soil organic matter: Destabilization of deeper soil carbon following harvesting of temperate coniferous forest. J Geophys Res. 2009;114:1-9.

Ellert BH, Bettany JR. Calculation of organic matter and nutrients stored in soils under contrasting management regimes. Can J Soil Sci. 1995;75:529-38.

Empresa Brasileira de Pesquisa Agropecuária - Embrapa. Sistema brasileiro de classificação de solos. $3^{\mathrm{a}}$ ed. Rio de Janeiro; 2013.

Freixo AA, Canellas LP, Machado PLOA. Propriedades espectrais da matéria orgânica leve-livre e leve intra-agregados de dois Latossolos sob plantio direto e preparo convencional. R Bras Ci Solo. 2002a;26:445-53.

Freixo AA, Machado PLOA, Dos Santos HP, Silva CA, Fadigas FS. Estoques de carbono e nitrogênio e distribuição de frações orgânicas de Latossolo do cerrado sob diferentes sistemas de cultivo. R Bras Ci Solo. 2002b;26:425-34

Golchin A, Oades JM, Skjemstad JO, Clarke P. Soil-structure and carbon cycling. Aust J Soil Res. 1994a;32:1043-68.

Golchin A, Oades JM, Skjemstad JO, Clarke P. Study of free and occluded particulate organic-matter in soils by solid-state ${ }^{13} \mathrm{C}$ CP/MAS NMR-Spectroscopy and scanning electron-microscopy. Aust J Soil Res. 1994b;32:285-309.

Hofmockel KS, Zak DR, Moran KK, Jastrow JD. Changes in forest soil organic matter pools after a decade of elevated $\mathrm{CO}_{2}$ and $\mathrm{O}_{3}$. Soil Biol Biochem. 2011;43:1518-27.

Inda-Junior AV, Bayer C, Conceição PC, Boeni M, Salton JC, Tonin AT. Variáveis relacionadas à estabilidade de complexos organo-minerais em solos tropicais e subtropicais brasileiros. $\mathrm{Ci}$ Rural. 2007;37:1301-7.

Johanson KJ. Iodine in soils. Svensk Kärnbränslehantering AB (SKB), Technical report TR-00-21 [internet]. Stockholm (Sweden); 2000 [acesso em 03 jul 2014]. Disponível em: www.skb.se/upload/ publications/pdf/TR-00-21.pdf. 
Kaiser K, Mikutta R, Guggenberger G. Stability of organic matter sorbed to porous ferrihydrite and goethite incrises with residence time. Soil Sci Soc Am J. 2007;71:711-9.

Kleber M, Nico PS, Plante AF, Filley T, Kramer M, Swanston C, Sollins P. Old and stable soil organic matter is not necessarily chemically recalcitrant: Implications for modeling concepts and temperature sensitivity. Global Change Biol. 2011;17:1097-107.

Moni C, Derrien D, Hatton PJ, Zeller B, Kleber M. Density fractions versus size separates: does physical fractionation isolate functional soil compartments? Biogeosci Disc. 2012;9:8405-47.

Morgun EG, Makarov MI. Use of sodium polytungstate in the granulo-densimetric fractionation of soil material. Eur Soil Sci. 2011;44:394-8.

Moulin V, Reiller P, Amekraz B, Moulin C. Direct characterization of iodine covalently bound to fulvic acids by eletrospray mass spectrometry. Rapid Commun Mass Spectrom. 2001;15:2488-96.

Paré MC, Bedard-Haughn A. Optimum liquid density in separation of the physically uncomplexed organic matter in Arctic soils. Can J Soil Sci. 2011;91:65-8.

Parfitt RL, Theng BKG, Whitton JS, Shepher TG. Effects of clay minerals and land use on organic matter pools. Geoderma. 1997;75:1-12.

Potes ML, Dick DP, Santana GS, Tomazi M, Bayer C. Soil organic matter in fire-affected pastures and in an Araucaria forest in South-Brazilian Leptosols. Pesq Agropec Bras. 2012;47:707-15.

Radlinger G, Heumann KG. Transformation of iodide in natural and wastewater systems by fixation on humic substances. Environ Sci Technol. 2000;34:3932-6

Roscoe R, Buurman P, Velthorst EJ, Vasconcellos CA. Soil organic matter dynamics in density and particle size fractions as revealed by the ${ }^{13} \mathrm{C} /{ }^{12} \mathrm{C}$ isotopic ratio in a Cerrado's Oxisol. Geoderma. 2001;104:185-202.

Rumpel C, Kögel-Knabner I. Deep soil organic matter - a key but poorly understood component of terrestrial C cycle. Plant Soil. 2011;338:143-58.
Schlegel ML, Reiller P, Mercier-Bion F, Barré N, Moulin V. Molecular environment of iodine in naturally iodinated humic substances: Insight from X-ray absorption spectroscopy. Geochim Cosmochim Acta. 2006;70:5536-51.

Shi XM, Li XG, Long RJ, Singh BP, Li ZT, Li FM. Dynamics of soil organic carbon and nitrogen associated with physically separated fractions in a grassland-cultivation sequence in the Qinghai-Tibetan plateau. Biol Fertil Soils. 2010;46:103-11.

Sohi SP, Mahieu N, Arah JRM, Powlson DS, Madari B, Gaunt JL. A procedure for isolating soil organic matter fractions suitable for modeling. Soil Sci Soc Am J. 2001;65:1121-8.

Sollins P, Homann P, Caldwell BA. Stabilization and destabilization of soil organic matter: mechanisms and controls. Geoderma. 1996;74:65-105.

Sun B, Hallett PD, Caul S, Daniell TJ, Hopkins DW. Distribution of soil carbon and microbial biomass in arable soils under different tillage regimes. Plant Soil. 2011;338:17-25.

Tomazi M, Bayer C, Conceição PC. O uso de amostras peneiradas a dois milímetros subestima a proteção física da matéria orgânica no solo. R Bras Ci Solo. 2011;35:1323-9.

Vieira FCB, Bayer C, Zanatta JA, Mielniczuk J, Six J. Building Up organic matter in a subtropical Paleudult under Legume cover-crop-based rotations. Soil Sci Soc Am J. 2009;73:1699-706.

Von Lützow M, Kögel-Knabner I. Temperate sensitivity of soil organic matter decomposition - what do we know? Boil Fertil Soils. 2009;46:1-15.

Wagai R, Mayer LM, Kitayama K. Nature of the "occluded" low density fraction in soil organic matter studies: a critical review. Soil Sci Plant Nutr. 2009;55:13-25.

Yamaguchi N, Nakano M, Takamatsu R, Tanida H. Inorganic iodine incorporation into soil organic matter: Evidence from iodine K-edge X-ray absorption near-edge structure. J Environ Radioact. 2010;101:451-7. 